OPEN ACCESS

Edited by: Micaela Sgorbini, University of Pisa, Italy

Reviewed by: Andrea Marchegiani, University of Camerino, Italy Roberta Perego,

University of Milan, Italy

*Correspondence: Ricardo J. Soares Magalhães r.magalhaes@uq.edu.au

Specialty section:

This article was submitted to Comparative and Clinical Medicine,

a section of the journal

Frontiers in Veterinary Science

Received: 17 December 2020 Accepted: 21 January 2021

Published: 15 February 2021

Citation:

Rynhoud H, Gibson JS, Meler E and Soares Magalhães RJ (2021) The Association Between the Use of Oclacitinib and Antibacterial Therapy in Dogs With Allergic Dermatitis: A Retrospective Case-Control Study. Front. Vet. Sci. 8:631443. doi: 10.3389/fvets.2021.631443

\section{The Association Between the Use of Oclacitinib and Antibacterial Therapy in Dogs With Allergic Dermatitis: A Retrospective Case-Control Study}

\author{
Hester Rynhoud ${ }^{1}$, Justine S. Gibson ${ }^{2}$, Erika Meler ${ }^{2}$ and Ricardo J. Soares Magalhães ${ }^{1,3 *}$ \\ ${ }^{1}$ UQ Spatial Epidemiology Laboratory, School of Veterinary Science, The University of Queensland, Gatton, QLD, Australia, \\ ${ }^{2}$ School of Veterinary Science, The University of Queensland, Gatton, QLD, Australia, ${ }^{3}$ Children Health and Environment \\ Program, Child Health Research Centre, The University of Queensland, South Brisbane, QLD, Australia
}

Background: Canine allergic dermatitis, including atopic dermatitis, often requires antibacterial therapy for concurrent infections. Oclacitinib is indicated for treatment of pruritus associated with allergic dermatitis and the clinical manifestations of atopic dermatitis in dogs aged $\geq 12$ months.

Hypothesis/Objectives: We aimed to determine if there was a quantitative difference in antibacterial use by dogs with allergic dermatitis receiving oclacitinib vs. other anti-pruritic therapies and before vs. after oclacitinib.

Animals: In this retrospective case-control study, cases $(n=58)$ included dogs suffering from allergic dermatitis aged $\geq 12$ months receiving oclacitinib and controls $(n=205)$ were counterpart dogs treated with other anti-pruritic therapies.

Methods: Clinical histories of dogs with allergic dermatitis were collected from a small animal university hospital. Multivariable logistic regression models were developed adjusting for underlying skin or ear conditions to determine whether cases were prescribed fewer antibacterials than controls.

Results: The odds of systemic antibacterial usage were lower in cases vs. controls [odds ratio (OR): 0.29 (95\% confidence interval 0.12-0.71); $P=0.007$ ]. The odds of amoxycillin clavulanic acid usage (12.5-25 mg/kg orally every $12 \mathrm{~h}$ ) was lower in cases vs. controls [OR: 0.08 (0.01-0.71); $P=0.024]$. Topical antibacterial drug use was reduced overall; however, only the odds of neomycin use was lower in cases vs. controls [OR: 0.3 (0.1-0.89); $P=0.029$ ]. Cases had higher odds of experiencing improvements in allergic dermatitis categories vs. controls [OR: 7.89 (3.26-19.13); $P<0.001$ ].

Conclusions and Clinical Importance: Our results suggest that use of oclacitinib to treat allergic dermatitis in dogs is associated with less antibacterial use than other anti-pruritic therapies.

Keywords: oclacitinib, allergic dermatitis, antibacterial, anti-pruritic, dogs 


\section{INTRODUCTION}

Dogs with allergic dermatitis, including atopic dermatitis, contact allergy, flea allergy dermatitis, and cutaneous adverse food reactions, present to veterinary clinics with the characteristic clinical sign of pruritus which is often associated with gross inflammation and secondary skin infections (1). Environmental triggers for acute flares of atopic dermatitis can include food, pollens, dust mites, and flea or other insect bites (2). Atopic dermatitis is a common allergic skin disease of dogs which can negatively influence pet and owner quality of life; therefore, effective treatment is necessary (3-6).

Treatment of allergic dermatitis aims to control pruritus and inflammation, as well as identify and eliminate underlying etiologies to prevent and reduce acute flares (2). Topical and systemic glucocorticoids can be used to treat both acute flares and chronic allergic dermatitis, respectively (2). Longterm glucocorticoid therapy is no longer recommended due to serious adverse effects, including gastrointestinal ulceration, hyperlipidaemia, diabetes mellitus, muscle wasting and iatrogenic hyperadrenocorticism (7). Systemic cyclosporin and topical tacrolimus can also be used to manage atopic dermatitis; however, the slow onset of action renders them inappropriate for acute flares (2). Lokivetmab, is also available to treat atopic dermatitis which has recently been reported to have a more pronounced effect on pruritis compared to cyclosporin (8). Antihistamines are also often used but are of limited to no benefit for dogs with atopic dermatitis (2). In addition, the use of non-irritating shampoos and oral essential fatty acid supplements has been suggested to support skin barrier function $(2,9)$. Alleviating pruritus is important when treating allergic dermatitis to interrupt the itch cycle and allow for skin healing, thus reducing chronic inflammatory changes and secondary infections (10).

Dogs with allergic dermatitis frequently have concurrent infections of the skin and ears $(9,11)$. Atopic dogs have a higher abundance of Staphylococcus spp. on their skin, notably Staphylococcus pseudintermedius, which is commonly implicated in pyoderma (12-14). Studies infer that staphylococci have increased adherence to inflamed and atopic skin, which could explain the increased abundance of this organism $(15,16)$. It has been suggested that these bacteria are also involved in hypersensitivity responses, most commonly due to staphylococcal components or toxins acting as superantigens (13). Treatment often requires topical and/or systemic antibacterial therapy (11). With increasing reports of bacterial resistance to antimicrobial therapy in humans and animals, veterinarians are encouraged to follow antimicrobial stewardship (AMS) guidelines when treating these cases to improve efficacy and limit further development of resistance $(2,17)$.

One way to aid AMS is to use treatments that reduce the need for antibacterials when treating allergic skin disease. Oclacitinib, a Janus kinase (JAK) inhibitor, targets specific pathways for cytokines involved in itch and inflammation (18). The JAK 1 enzyme is involved in signaling and signal transduction of proinflammatory, pro-allergic and pruritogenic cytokines associated with atopic dermatitis (10). Oclacitinib inhibits predominantly JAK 1 dependent cytokines and effectively treats the clinical signs associated with allergic dermatitis and atopic dermatitis in dogs (18).

The primary aim of this retrospective study was to quantify changes in systemic and topical antibacterial use in clientowned dogs with allergic dermatitis, including atopic dermatitis, following administration of oclacitinib compared to dogs that were not prescribed oclacitinib, and also before and after initial oclacitinib use. The effects of oclacitinib on corticosteroid use and skin conditions were secondary objectives.

\section{MATERIALS AND METHODS}

\section{Ethical Statement}

The data used were originally collected by veterinarians undertaking standard veterinary practices, therefore animal ethics approval was not required. Animals were deidentified during the data collection process for this study and data was stored and analyzed following the University of Queensland's research integrity protocols.

\section{Case Definitions and Data Sources}

All dogs included in the retrospective case-control study were $\geq 12$ months old in accordance with the minimum approved age for administration of oclacitinib. Cases and controls were initially identified using specific search terms as listed in Supplementary Table 1. Only on label conditions were included in the initial search terms. Dogs were conditionally diagnosed with an allergic skin condition if a definitive diagnoses was not provided. Diagnostic tests performed to rule out other causes of itchy skin included ear smears, flea combs, skin scrapes, sticky tape, and cultures; however, not all consultation notes had record of these tests.

Inclusion criteria for cases included dogs diagnosed with an allergic skin condition (atopic dermatitis, contact allergy, flea allergy dermatitis, cutaneous adverse food reactions) that were treated with oclacitinib. Cases were identified in the veterinary clinical database between January 2016 and July 2018 in accordance with oclacitinib's release to market in Australia in early 2016. Medical history was collected from the date of initial diagnosis with an allergic skin condition. In total, consultation data for cases were sourced between November 2010 and July 2018, allowing case data to be classified into "before oclacitinib" and "after oclacitinib."

Inclusion criteria for controls included dogs diagnosed with an allergic skin condition that were not treated with oclacitinib. Allergic dermatitis, including atopic dermatitis, is often managed multimodally, therefore other therapeutics were frequently used in the management of these dogs. Controls were identified in the clinical database as dogs that never received oclacitinib, with data collected from clinic consultations between January 2010 and July 2018. The consultations included in the analysis also counted medication dispensations (where consultation notes only included medication dispensations for both cases and controls). 
Data were extracted from the veterinary clinical database at a university small animal teaching hospital. Demographic data collected included signalment, breed, size, and reproductive status. Dogs were grouped in categories according to size as small, medium or large, based on Pickup et al. (19) who used these categories according to the UK Kennel Club classification (20). Clinical history data included consultation type, date, clinical signs, diagnostic tests, diagnosis, treatment plans, drugs administered, dosage and dose frequencies and outcome of most recent consultation. Antibacterials were divided into topical and systemic which included agents used for cutaneous and otic conditions. Antibacterials included antiseptics and biocides. Every time a drug, including oclacitinib, was prescribed and dispensed in a consultation this was defined as a "course." A detailed description of the data used is available in Supplementary Table 2 .

\section{Statistical Analyses Descriptive Analysis}

Statistical analyses involved comparisons between case (dogs treated with oclacitinib) and control data and within case data before and after initial oclacitinib use. Topical and systemic antibacterial usage was depicted as total number of courses prescribed per dog for all consultations recorded in the study. All statistical analyses comparing cases and controls (including regression models) used case data post-oclacitinib. Pre-oclacitinib data was only used when comparing before vs. after oclacitinib use within cases. The total timespan for the cases both pre- and post-oclacitinib use was November 2011 to July 2018. The timespan for cases post-oclacitinib use was from March 2016 to July 2018. The distribution of the number of antibacterial courses per dog was not normally distributed and so non-parametric tests were used. To detect whether the use of topical and systemic antibacterials differed significantly between cases and controls, a Wilcoxon rank-sum test was conducted. To determine whether there was a significant difference in topical and systemic antibacterial use before and after initial oclacitinib use in cases, a Wilcoxon signed-rank test was performed. Similar tests were performed to determine differences between total number of courses per dog in small, medium and large breeds. To determine if the number of courses of oclacitinib influenced antimicrobial use within the cases, a Kruskal-Wallis test was performed to identify whether there were significant differences in antimicrobial use within cases based on the number of oclacitinib courses they received (one course, two courses, or three or more courses). A chi-square test was used to determine if the proportional use of systemic and topical antibacterials differed significantly between the cases and controls and before and after oclacitinib use in cases. All descriptive statistical analyses were performed using Microsoft Excel 2016 and Stata version 13.1 (Stata Corporation, College Station, TX, USA).

\section{Models Adjusted for Overall Drug Use and Specific Drug Groups}

Bernoulli logistic regression models were developed with oclacitinib treated dogs (cases) as the outcome of interest and dogs not treated with oclacitinib as the reference group; this approach models the probability of being a case. The predictor variables used in the logistic regression models are listed in Supplementary Table 3. Analysis was conducted in two phases. First, the univariable association between cases and different drug therapies was evaluated using univariable logistic regression. A cut off $P$-value of $\leq 0.20$ was used when selecting predictor variables to be included in the full multivariable model. Pearson's correlation coefficient was used to test for correlation between variables with a $P$-value $\leq 0.20$. If a pair of variables had a correlation coefficient $>0.8$ then only one was considered in the full multivariable model. Second, variables that were significant in the univariable analysis were included in a full multivariable model. The final multivariable model was arrived at using a manual backward stepwise variable selection process. Confounding variables were identified by assessing the impact of variable removal on the coefficients of the remaining variables. If the coefficient of one variable changed by $>25 \%$ when a variable was eliminated, then it was considered a confounder and put back into the multivariable model. Drugs included in the final multivariable model with a $P$-value of $\leq 0.05$ were termed significant in this study.

Two separate multivariable logistic regression models were considered. The first multivariable model quantified the relationship between cases (oclacitinib treated dogs) and controls and overall drug use (Model 1). A second multivariable model quantified differences between cases and controls with regards to individual drug data, specifically different doses and mean number of courses per animal for an individual drug (Model 2). This was achieved by including the total number of courses per animal for total antibacterial, glucocorticoid and other drug use (including antihistamines and cyclosporin) for all animals. Due to the significant level of missing data (i.e., 38\% in cases and 32\% in controls) the length (i.e., duration in days) of antibacterial courses was not included in our models.

\section{Models Adjusted for Type of Skin Condition and Presence of An Ear Condition}

To determine if skin and ear conditions influenced antibacterial use in oclacitinib treated dogs (cases) and controls, Model 1 and Model 2 were divided into four sub models. Firstly, skin condition categories and changes in these categories were included in two sub models. Skin conditions were allocated to three categories; allergic dermatitis without secondary infection, allergic dermatitis with secondary superficial bacterial pyoderma and allergic dermatitis with secondary deep bacterial pyoderma (Supplementary Table 6). Changes were recorded if there were variations in skin condition category after initial consultations where oclacitinib was prescribed in cases and not prescribed in controls. Given the time-series nature of our database we constructed an explanatory variable that captures the dynamic nature without overfitting the model with temporal lags; as a result the proxy variable to measure skin condition changes in dogs was used to determine whether there was a general positive or negative response after treatment with oclacitinib.

The first sub model adjusted drug effects for baseline skin condition category (Model A) and the second sub model adjusted drug effects for changes in skin condition category for individuals 
diagnosed with a skin condition at the initial consultation (Model B). Individuals that experienced ear conditions instead of skin conditions were omitted from Model B. A further two sub models were created to determine whether the presence of ear conditions and changes in causative infectious agents influenced drug use in cases and controls. Ear condition was used as an overarching term that included all pruritic ears that could be both infected and uninfected. The ear condition variable was divided into seven categories. These included pruritic ears without the presence of an infectious agent, presence of cocci, presence of rods, presence of gram positive bacteria, presence of gram negative bacteria, a ruptured tympanic membrane and presence of Malassezia pachydermatis (Supplementary Table 6). The third sub model adjusted drug effects for the presence or absence of an ear condition (Model C) and the fourth model adjusted drug effects for the changes between infectious agents isolated from ears for individuals that experienced ear conditions (Model D).

Interaction terms between skin condition category and changes between skin condition categories and ear conditions and changes between infectious agents were also tested but due to the low number of events for some variables (Supplementary Tables 7, 8) we reported fixed effect logistic regression models only. All multivariable statistical analyses were performed using the statistical software Stata version 13.1.

\section{RESULTS}

\section{Dataset for Analysis}

The search for clinical records resulted in a one to four ratio for dogs treated with oclacitinib (cases $=58$ ) and dogs treated with other therapeutics (controls $=205$ ). The distribution of sex, neuter status, age, and breed are displayed in Table 1. General practice consultations represented $93 \%$ of cases and $90 \%$ of controls. Dermatology specialist consultations accounted for $1 \%$ of visits for both groups. The remaining consultation types included internal medicine, surgical or hospitalized patients. The proportions of small, medium and large breeds were evenly distributed in cases and controls. There were more small breeds in both groups. Small breeds accounted for half of the cases and controls, where medium and large breeds each accounted for 21-25\% of cases and controls (Table 1). Information on the length of courses in days for each antibiotic is available in Supplementary Table 9. The majority of dogs were treated with antibiotics empirically with only 16 (4 cases and 12 controls) having culture and susceptibility tests performed. Once test results were available dogs were treated with an appropriate antibiotic (i.e., one that for which the isolated bacteria was susceptible).

\section{Descriptive Results \\ Distribution of Antibacterial Use in Dogs Treated With Oclacitinib (Cases) vs. Dogs Not Treated With Oclacitinib (Controls)}

Total antibacterial use is shown in Figure 1. Both dogs treated with oclacitinib (cases) and dogs not treated with oclacitinib (controls) were prescribed a higher proportion of topical than systemic antibacterials. Cephalexin courses constituted $94 \%$
TABLE 1 | Demographic data of dogs for cases and controls.

\begin{tabular}{|c|c|c|}
\hline Variables & Cases & Controls \\
\hline Total & 58 & 205 \\
\hline \multicolumn{3}{|l|}{ Sex } \\
\hline Male & 28 (48\%) & $100(49 \%)$ \\
\hline Female & $30(52 \%)$ & 105 (51\%) \\
\hline \multicolumn{3}{|c|}{ Neuter status } \\
\hline Neutered & 48 (83\%) & $150(73 \%)$ \\
\hline Entire & $10(17 \%)$ & 55 (27\%) \\
\hline \multicolumn{3}{|c|}{ Age (years) } \\
\hline $1-4$ & 22 (38\%) & 77 (38\%) \\
\hline$>4-8$ & $21(36 \%)$ & 77 (38\%) \\
\hline$>8$ & $15(26 \%)$ & $51(24 \%)$ \\
\hline \multicolumn{3}{|l|}{ Breed } \\
\hline Small & $29(50 \%)$ & $110(54 \%)$ \\
\hline Medium & $14(24 \%)$ & 44 (21\%) \\
\hline Large & 15 (25\%) & $51(24 \%)$ \\
\hline
\end{tabular}

Supplementary Tables $4 a, b$ summarize the distribution of breeds in the three groups based on (19).

(17/18) of systemic antibacterial courses in the cases after initial oclacitinib use and $72 \%(125 / 173)$ in the controls. Amoxycillin clavulanic acid comprised 6\% (1/18) of systemic antibacterial courses in the cases after initial oclacitinib use and $28 \%(48 / 173)$ in the controls. There were no significant differences in proportional use of systemic antibacterials or topical antibacterials in the cases and controls $(P=0.98)$.

There were twice as many topical and systemic antibacterial courses in total per animal in controls [total: mean (95\% CI) $2.7(2.27-3.21)]$ than in cases $[1.3(0.93-1.66)](P<$ 0.001) (Figure 2). The difference between the total number of topical $(P=0.019)$ or systemic $(P<0.001)$ antibacterial courses per animal in the cases vs. controls was also significant. Cases had a significantly $(P=0.016)$ lower total number of cephalexin courses $[0.3(0.15-0.44)]$ per animal than controls $[0.6(0.49-0.73)]$. Cases also had a significantly $(P=0.002)$ lower total number of amoxycillin clavulanic acid courses per animal $[0.01(-0.02-0.05)]$ than controls [0.2 (0.16-0.31)] (Supplementary Figure 1).

Controls had higher mean numbers of courses of topical antibiotics such as chlorhexidine, salicylic acid, polymyxin B, neomycin, fusidic acid and ciprofloxacin (Supplementary Figure 3). It is important to note that polymixin B, enrofloxacin and ciprofloxacin are considered as Highest Priority Critically Important Antimicrobials (21). While cases had more courses of florfenicol (Supplementary Figure 3), actual use of this drug was extremely low in the study population. None of the differences in topical treatments between cases and controls were statistically significant.

The mean number of topical or systemic courses per animal was lower in cases vs. controls for small, medium and large breeds (Supplementary Figures 5, 7, 9). In small breeds, this difference for cases vs. controls was significant for both topical $(P=0.016)$ and systemic $(P<0.001)$ antibacterials, however there was no 


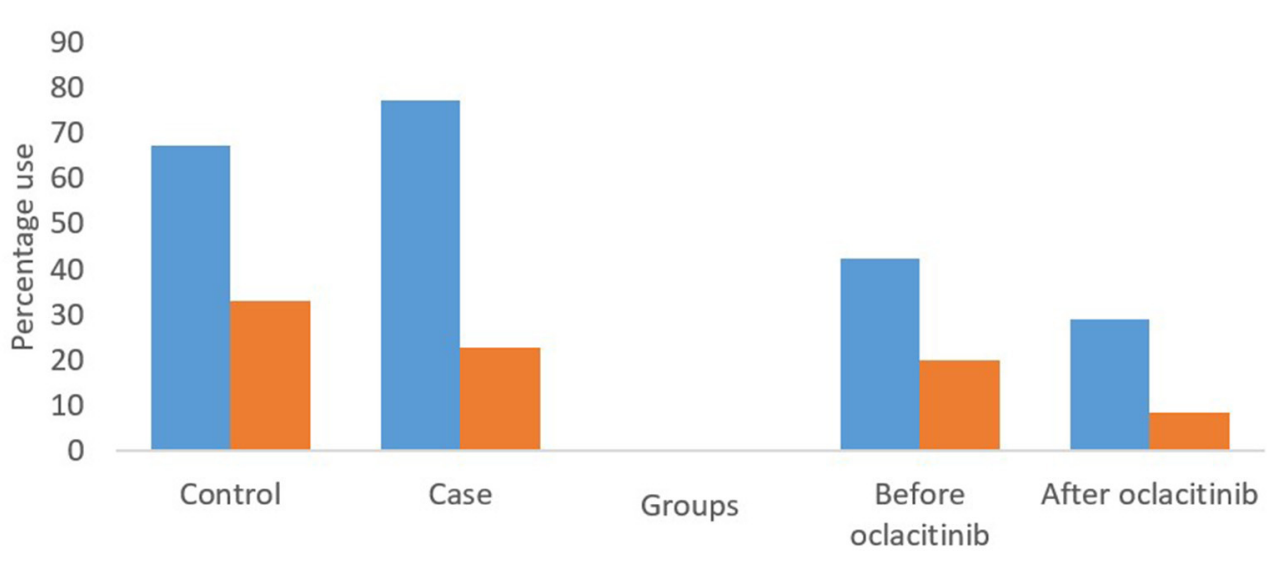

Topical antibacterials

- Systemic antibacterials

FIGURE 1 | Systemic and topical antibacterial use in 58 cases and 205 controls with allergic dermatitis for all consults included in the study. The first two sets of bars represent the percentages of topical and systemic antibacterial courses prescribed in cases after oclacitinib use and controls. The final two sets of bars represent the percentages of topical and systemic antibacterial prescribed in cases before and after oclacitinib.

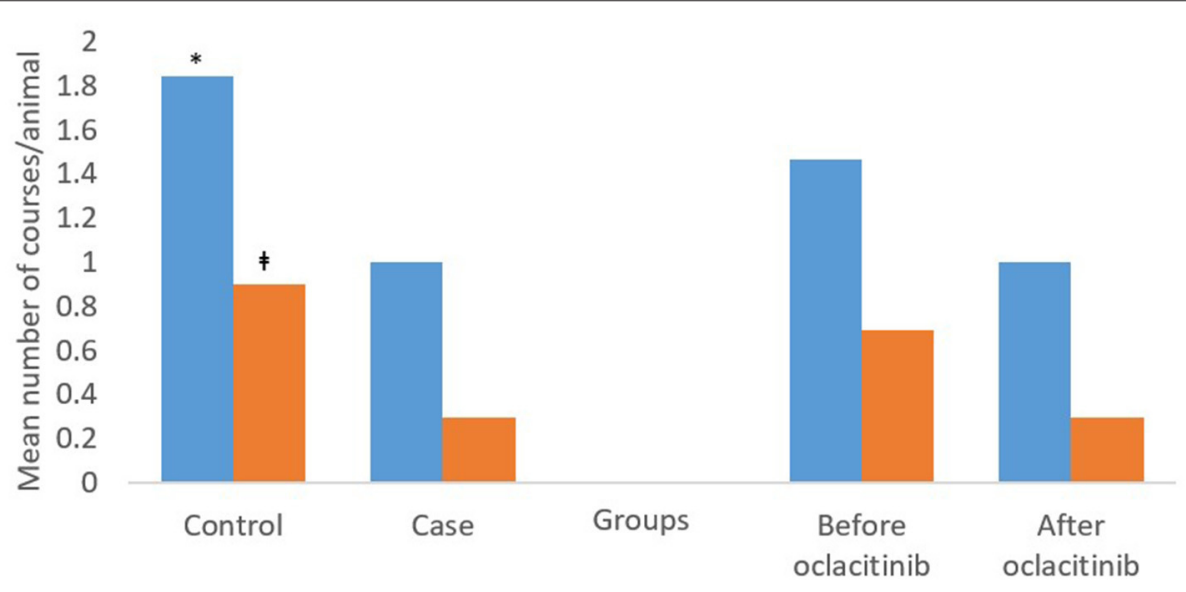

Topical antibacterials $\quad$ Systemic antibacterials

FIGURE 2 | The mean topical and systemic antibacterial courses per animal in 58 cases and 205 controls, and before and after initial oclacitinib use in 58 cases for all consults included in the study. ${ }^{\star} P=0.019$ vs. cases; ${ }^{\ddagger} P<0.001$ vs. cases. The first two sets of bars represent the percentages of topical and systemic antibacterial courses prescribed in cases after oclacitinib use and controls. The final two sets of bars represent the percentages of topical and systemic antibacterial prescribed in cases before and after oclacitinib.

significant difference between cases and controls in medium or large breeds.

No significant differences were identified in total antimicrobial use $(p=0.7094)$, total topical antimicrobial use $(p=0.5852)$, or total systemic antimicrobial use $(p=0.7509)$ between dogs that received different amounts of oclacitinib courses (one, two or three or more).

\section{Distribution of Antibacterial Use Before vs. After Oclacitinib Use Within Cases}

Topical antibacterials comprised a higher proportion of total antibacterials prescribed, both before and after oclacitinib use in cases (Figure 1). There were no significant differences in the proportional use of systemic or topical antibacterials $(P=$ $0.201)$ before or after oclacitinib use. Sixty-one percent $(27 / 44)$ of cephalexin courses were administered before initial oclacitinib use and $88 \%(7 / 8)$ of the amoxycillin clavulanic acid courses prescribed for cases were prescribed before initial oclacitinib use. There was no statistically significant differences between these proportions $(P=0.305)$.

The mean $[95 \% \mathrm{CI}]$ number of total antibacterial courses (systemic plus topical) per animal was higher before [2.2 $(1.32-3)]$ than after $[1.3(0.93-1.66)]$ initial oclacitinib use. The difference between total number of topical or systemic 
TABLE 2 | The univariable results of associations between cases and controls with respect to signalment, drug therapy and, skin or ear conditions.

\begin{tabular}{|c|c|c|c|}
\hline Variable & $\begin{array}{l}\text { Odds ratio (95\% } \\
\text { confidence } \\
\text { interval) }\end{array}$ & $P$ value & $\begin{array}{l}\text { Overall } P \\
\text { value }\end{array}$ \\
\hline Age (years) & & & 0.980 \\
\hline $1-4$ & Reference & & \\
\hline$>4-8$ & $0.95(0.49-1.88)$ & 0.893 & \\
\hline$>8$ & $1.03(0.49-2.17)$ & 0.939 & \\
\hline \multicolumn{4}{|l|}{ Sex } \\
\hline Male & Reference & & \\
\hline Female & $1.02(0.57-1.83)$ & 0.946 & \\
\hline Breed $^{\square}$ & & & 0.870 \\
\hline Small & Reference & & \\
\hline Medium & $1.21(0.58-2.5)$ & 0.612 & \\
\hline Large & $1.12(0.55-2.26)$ & 0.761 & \\
\hline \multicolumn{4}{|l|}{ Neuter } \\
\hline Neutered & Reference & & \\
\hline Entire & $0.57(0.27-1.2)$ & 0.139 & \\
\hline $\begin{array}{l}\text { Type of skin condition } \\
\text { category at baseline }\end{array}$ & & & $<0.001$ \\
\hline $\begin{array}{l}\text { Non-bacterial allergic } \\
\text { dermatitis }\end{array}$ & Reference & & \\
\hline No skin condition & $0.73(0.61-0.89)$ & 0.001 & \\
\hline Superficial pyoderma & $0.84(0.76-0.94)$ & 0.001 & \\
\hline \multicolumn{4}{|c|}{ Changes in skin condition category } \\
\hline No change & Reference & & \\
\hline $\begin{array}{l}\text { Improvement in skin } \\
\text { condition category }\end{array}$ & $1.25(1.13-1.4)$ & $<0.001$ & \\
\hline \multicolumn{4}{|l|}{ Ear condition } \\
\hline No ear condition & Reference & & \\
\hline Ear condition present & $0.65(0.34-1.23)$ & 0.183 & \\
\hline \multicolumn{4}{|c|}{ Changes between infectious agents present in ears } \\
\hline No change & Reference & & \\
\hline Change present & $0.51(0.17-1.53)$ & 0.228 & \\
\hline \multicolumn{4}{|l|}{ Total number of courses } \\
\hline All antibacterials & $0.72(0.59-0.88)$ & 0.001 & \\
\hline Topical antibacterials & $0.78(0.63-0.96)$ & 0.02 & \\
\hline Systemic antibacterials & $0.4(0.24-0.66)$ & $<0.001$ & \\
\hline All glucocorticoids & $0.46(0.33-0.64)$ & $<0.001$ & \\
\hline Topical glucocorticoids & $0.54(0.37-0.78)$ & 0.001 & \\
\hline Systemic glucocorticoids & $0.3(0.17-0.54)$ & $<0.001$ & \\
\hline $\begin{array}{l}\text { Other (antihistamines and } \\
\text { cyclosporin) }\end{array}$ & $0.4(0.14-1.14)$ & 0.086 & \\
\hline \multicolumn{4}{|c|}{ Number of courses of antibacterials } \\
\hline $\begin{array}{l}\text { Chlorhexidine unknown } \\
\text { frequency }\end{array}$ & $0.17(0.02-1.26)$ & 0.082 & \\
\hline Chlorhexidine $^{*}$ & $0.2(0.03-1.41)$ & 0.106 & \\
\hline Cephalexin & $0.64(0.4-1.03)$ & 0.069 & \\
\hline Neomycin* & $0.32(0.13-0.81)$ & 0.016 & \\
\hline Polymyxin $B^{*}$ & $0.68(0.45-1.04)$ & 0.077 & \\
\hline Amoxycillin clavulanic acid• & $0.13(0.02-0.89)$ & 0.038 & \\
\hline \multicolumn{4}{|c|}{ Number of courses of other drugs } \\
\hline Prednisolone acetate $^{\star}$ & $0.7(0.47-1.04)$ & 0.075 & \\
\hline Hydrocortisone ${ }^{\star}$ & $0.32(0.13-0.81)$ & 0.016 & \\
\hline
\end{tabular}

(Continued)
TABLE 2 | Continued

\begin{tabular}{llll}
\hline Variable & $\begin{array}{c}\text { Odds ratio (95\% } \\
\text { confidence } \\
\text { interval) }\end{array}$ & $\boldsymbol{P}$ value & $\begin{array}{c}\text { Overall } \boldsymbol{P} \\
\text { value }\end{array}$ \\
\hline Prednisolone $\circ$ & $0.26(0.11-0.65)$ & 0.004 & \\
Prednisolone $\diamond$ & $2.05(0.74-5.73)$ & 0.169 \\
Chlorpheniramine & $0.22(0.03-1.44)$ & 0.114 & \\
\hline
\end{tabular}

${ }^{*}$ Topical antibacterial high frequency $\geq$ once per week $\diamond$ Systemic tapering dose 1.1-4 $\mathrm{mg} / \mathrm{kg} /$ day o Systemic tapering dose $0.5-1 \mathrm{mg} / \mathrm{kg} /$ day $\bullet$ Systemic dose $12.5-25 \mathrm{mg} / \mathrm{kg}$ every $12 \mathrm{~h}$.

-Systemic dose 15-30 mg/kg every $12 \mathrm{~h}$ 口Breed categories defined in Supplementary Tables 4a,b.

antibacterial courses per animal before vs. after initial oclacitinib use was not significant) (Figure 2). After initial oclacitinib use, the total number of amoxycillin clavulanic acid $(P$ $=0.048)$ and cephalexin courses $(P=0.336)$ per animal decreased (Supplementary Figure 2). While cases had higher mean numbers of courses for salicylic acid, neomycin, polymyxin B, florfenicol, enrofloxacin, and ciprofloxacin before (vs. after) oclacitinib use (Supplementary Figure 4), none of the differences were statistically significant.

Supplementary Figures 6, 8, 10 display the mean number of courses per breed group before and after initial oclacitinib use in the 58 cases. Both topical and systemic antibacterial use was more numerous in cases before initial oclacitinib use in small and medium breeds. Little to no difference was observed in large breeds. There was a significant difference in the number of systemic antibacterials used by small breeds before and after oclacitinib $(P=0.002)$. There were no significant differences in systemic or topical antibacterial use in medium or large breeds before or after oclacitinib use.

\section{Multivariable Logistic Regression Models Overall Antibacterial Usage in Dogs Treated With Oclacitinib (Cases) vs. Dogs Not Treated With Oclacitinib (Controls)}

Univariable analyses revealed that the total number of courses of antibacterials, glucocorticoids or "other" drug use had a significant effect on the outcome of being a case (Table 2). The odds of antibacterial use overall was lower in dogs treated with oclacitinib than controls [OR: $0.72(0.59-0.88) ; P=0.001]$. Similarly, the odds of topical [OR: $0.78(0.63-0.96) ; P=0.02$ ] and systemic [OR: $0.4(0.24-0.66) ; P<0.001]$ antibacterial use was lower in cases than controls. The odds of glucocorticoid use was also lower in cases than controls for overall [OR: 0.46 (0.33-0.64); $P<0.001$ ], topical [OR: $0.54(0.37-0.78) ; P=0.001$ ], and systemic [OR: $0.3(0.17-0.54) ; P<0.001]$ glucocorticoid use. The odds of "other" treatments were also lower in cases vs. controls [OR: $0.4(0.14-1.14) ; P=0.086]$. Initial logistic regression results suggested that the outcome of being a case was also significantly associated with the use of chlorhexidine, cephalexin, neomycin, polymyxin B, amoxycillin clavulanic acid, prednisolone acetate, hydrocortisone, systemic prednisolone and chlorpheniramine with the odds of the use of any of these agents being lower in cases than controls. 


\section{Effect of Skin and Ear Conditions in Dogs Treated With Oclacitinib (Cases) vs. Dogs Not Treated With Oclacitinib (Controls)}

Our univariable analyses indicate that after initial oclacitinib use, cases had lower odds of having no skin conditions [OR: $0.73(0.61-0.89) ; P=0.001]$, or superficial pyoderma [OR: $0.84(0.76-0.94) ; P=0.001]$ at initial consultation, compared to controls (Table 2). In addition, cases had significantly higher odds of experiencing improvement in skin condition category vs. controls [OR: $1.25(1.13-1.4) ; P<0.001]$. The majority of this improvement was dogs categorized with superficial pyoderma in the initial consult being categorized as a non-bacterial dermatitis due to allergic conditions at a subsequent consultation. Using this classification of change (changes earlier for models adjusted for type of skin condition and presence of ear condition), $48 \%$ of cases' skin conditions improved, $50 \%$ stayed the same and $2 \%$ worsened (Supplementary Table 5).

\section{Effect of Baseline Skin Condition and Changes in Condition on Overall Antibacterial Usage in Dogs Treated With Oclacitinib (Cases) vs. Dogs Not Treated With Oclacitinib (Controls)}

After accounting for age, sex, breed, neuter status and categorization of skin condition at baseline, the results of Model $1 \mathrm{~A}$ determined that the odds of systemic antibacterial use [OR: 0.4 (0.18-0.92); $P=0.031$ ], systemic glucocorticoid use [OR: 0.29 (0.15-0.56); $P<0.001]$ and use of other drugs (antihistamines and cyclosporin) [OR: $0.32(0.1-0.97) ; P=0.044$ ] was lower in cases than in controls. After adjusting for baseline skin condition category and changes in skin condition category, the results in Model 1B were similar [odds of systemic antibacterial [OR: 0.29 (0.12-0.71); $P=0.007$ ], glucocorticoid [OR: $0.29(0.15-0.57)$; $P<0.001$ ] and other drug [OR: $0.3(0.1-0.93) ; P=0.037]$ use was lower in cases]. Cases had higher odds of experiencing improvements in skin condition category vs. controls [OR: 7.89 (3.26-19.13); $P<0.001$ ] (Table 3).

TABLE 3 | Multivariable results displaying the association between case and controls with respect to overall drug use.

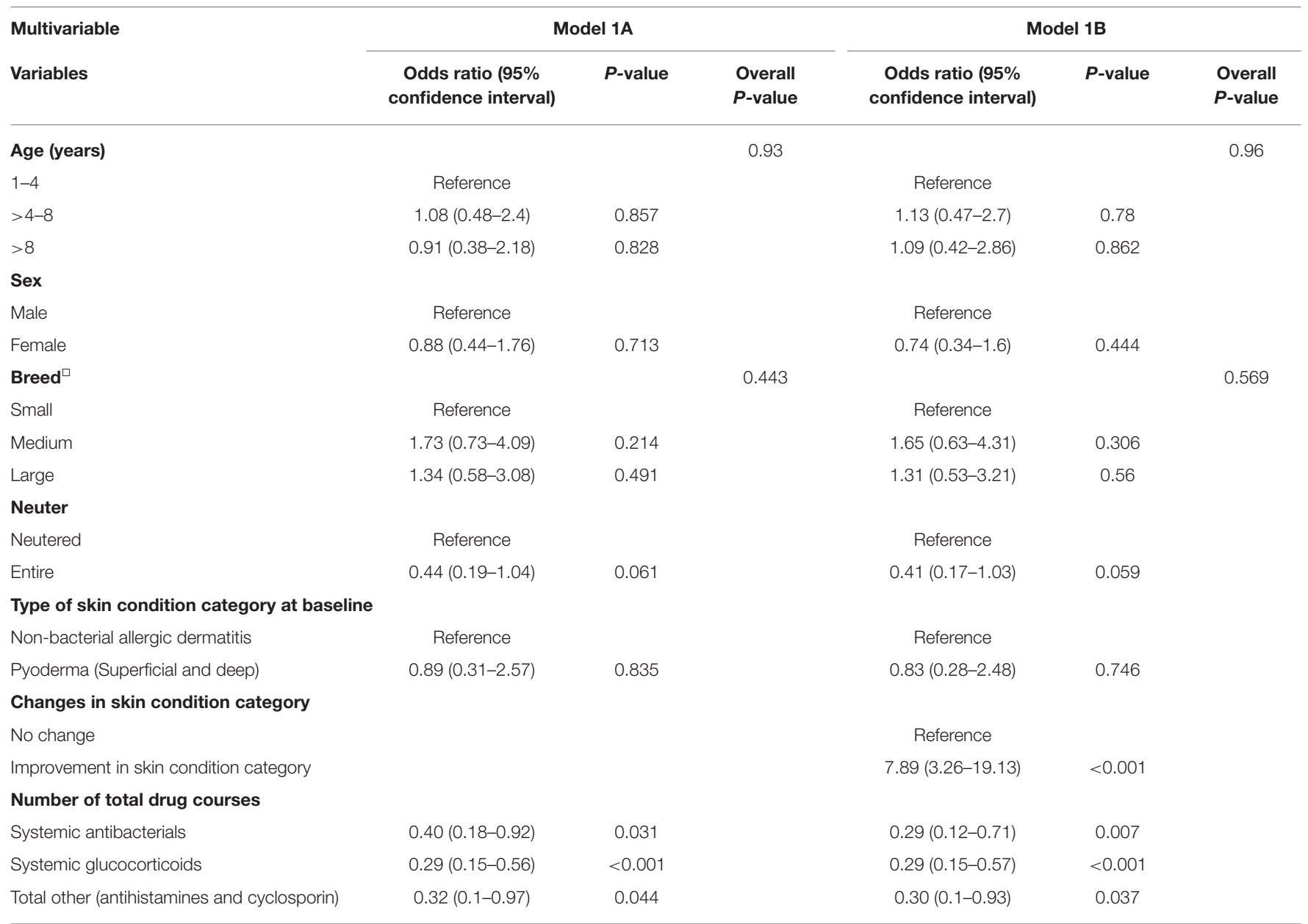

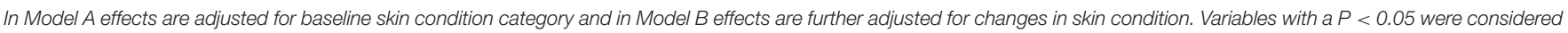
statistically significant. ${ }^{\square}$ Breed categories defined in Supplementary Tables $4 a, b$. 


\section{Effect of Individual Antibacterial Class and Glucocorticoid Usage Patterns in Dogs Treated With Oclacitinib (Cases) vs. Dogs Not Treated With Oclacitinib (Controls)}

After accounting for age, sex, breed, neuter status and type of skin condition at baseline, the results of Model 2A indicate that the odds of topical neomycin use [OR: $0.26(0.09-0.77) ; P=$ $0.015]$, amoxycillin clavulanic acid use [12.5-25 $\mathrm{mg} / \mathrm{kg}$ every $12 \mathrm{~h}$; OR: $0.12(0.01-1) ; P=0.050]$ and systemic prednisolone use [at a tapering dose of $0.5-1 \mathrm{mg} / \mathrm{kg} /$ day; OR: $0.23(0.09-$ $0.63) ; P=0.004$ ] were lower in cases compared to controls. After adjusting for baseline skin condition category and changes in skin condition category, the results in Model 2B were also similar (odds of use lower in cases vs. controls) for these doses of amoxycillin clavulanic acid [OR: $0.08(0.01-0.71) ; P$ $=0.024]$ and prednisolone [OR: $0.26(0.09-0.71) ; P=0.009$ ]. Neomycin was no longer retained as significant. Cases had much higher odds of experiencing improvements in skin condition category than controls [OR: $5.77 \quad(2.51-13.28) ; P<0.001]$ (Table 4).

\section{Effect of Ear Condition and Infectious Agents on Overall Antibacterial Usage in Dogs Treated With Oclacitinib (Cases) vs. Dogs Not Treated With Oclacitinib (Controls)}

After accounting for age, sex, breed, neuter status and presence of an ear condition, the results of Model 1C showed the odds of systemic antibacterial [OR: $0.44(0.25-0.77) ; P=0.004$ ] and systemic glucocorticoid [OR: $0.31(0.16-0.6) ; P<0.001$ ] use were lower in cases than controls (Table 5). After adjusting for the presence of an ear condition and the changes in causative infectious agents, the results in Model 1D revealed no significant association between cases and drug therapies.

\section{Effect of an Ear Condition and Infectious Agents Individual Antibacterial Class Usage Patterns in Dogs Treated With Oclacitinib (Cases) vs. Dogs Not Treated With Oclacitinib (Controls)}

After accounting for age, sex, breed, neuter status and presence of an ear condition, the results of Model 2C determined that

TABLE 4 | Multivariable results displaying the association between cases and controls with respect to specific drug types.

\begin{tabular}{|c|c|c|c|c|c|c|}
\hline $\begin{array}{l}\text { Multivariable } \\
\text { Variables }\end{array}$ & \multicolumn{3}{|c|}{ Model 2A } & \multicolumn{3}{|c|}{ Model 2B } \\
\hline Age (years) & & & 0.907 & & & 0.985 \\
\hline $1-4$ & Reference & & & Reference & & \\
\hline$>4-8$ & $1.2(0.54-2.68)$ & 0.659 & & $1.07(0.45-2.52)$ & 0.885 & \\
\hline Male & Reference & & & Reference & & \\
\hline Female & $1.05(0.52-2.09)$ & 0.894 & & $0.85(0.4-1.81)$ & 0.675 & \\
\hline Breed $^{\square}$ & & & 0.457 & & & 0.664 \\
\hline Small & Reference & & & Reference & & \\
\hline Medium & $1.73(0.7-4.26)$ & 0.231 & & $1.54(0.58-4.13)$ & 0.388 & \\
\hline Entire & $0.48(0.2-1.15)$ & 0.100 & & $0.42(0.16-1.08)$ & 0.073 & \\
\hline \multicolumn{7}{|c|}{ Type of skin condition category at baseline } \\
\hline Non-bacterial allergic dermatitis & Reference & & & Reference & & \\
\hline Pyoderma (Superficial and deep) & $0.56(0.21-1.51)$ & 0.253 & & $0.51(0.19-1.42)$ & 0.20 & \\
\hline \multicolumn{7}{|l|}{ Changes in skin condition category } \\
\hline No change & & & & Reference & & \\
\hline Improvement in skin condition category & & & & $5.77(2.51-13.28)$ & $<0.001$ & \\
\hline \multicolumn{7}{|l|}{ Number of total drug courses } \\
\hline Neomycin* & $0.26(0.09-0.77)$ & 0.015 & & & & \\
\hline Amoxycillin clavulanic acid• & $0.12(0.01-1)$ & 0.05 & & $0.08(0.01-0.71)$ & 0.024 & \\
\hline Prednisolone ○ & $0.23(0.09-0.63)$ & 0.004 & & $0.26(0.09-0.71)$ & 0.009 & \\
\hline
\end{tabular}

In Model A effects are adjusted for baseline skin condition category and in Model B effects are further adjusted for changes in skin condition. Variables with a P < 0.05 were considered statistically significant.

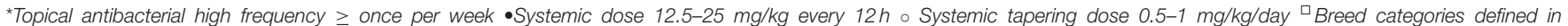
Supplementary Tables 4a,b. 
TABLE 5 | Multivariable results of the association between drug cases and controls with respect to overall drug use.

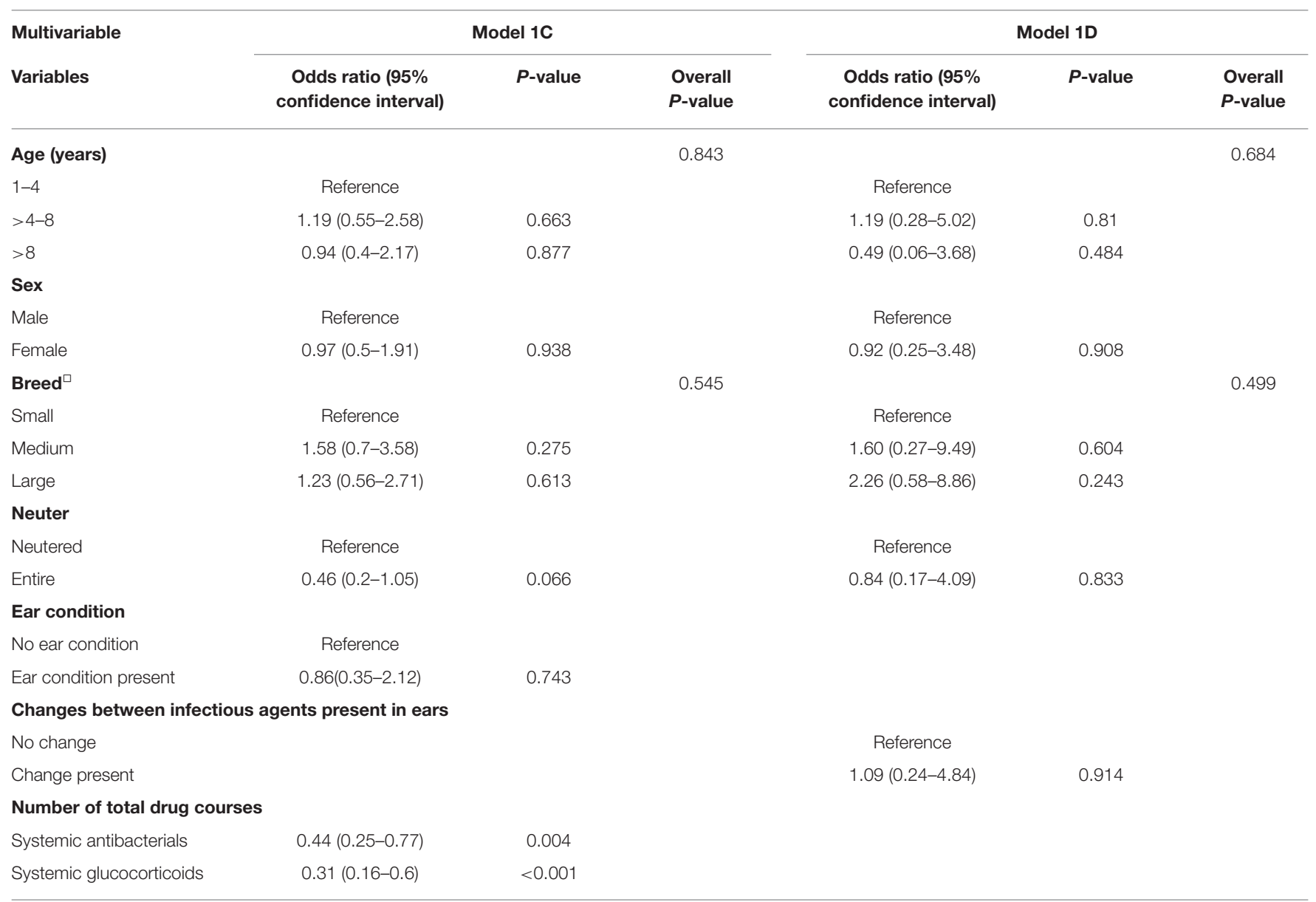

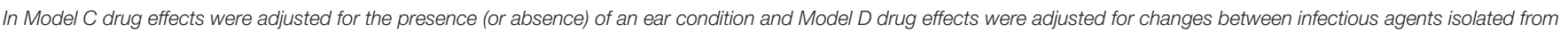
ears. Variables with a $P<0.05$ were significantly associated. ${ }^{\square}$ Breed categories defined in Supplementary Tables $4 a, b$.

the odds of neomycin use at high frequencies [OR: $0.3(0.1-$ $0.89) ; P=0.029]$, amoxycillin clavulanic acid use at $12.5-25$ $\mathrm{mg} / \mathrm{kg}$ every $12 \mathrm{~h}$ [OR: $0.1(0.01-0.83) ; P=0.032$ ] and systemic prednisolone use at a tapering dose of $0.5-1 \mathrm{mg} / \mathrm{kg} /$ day [OR: 0.25 (0.1-0.67); $P=0.006$ ] were lower in cases compared to controls (Table 6). After adjusting for the presence of an ear condition and the changes in causative infectious agents, the results in Model $1 \mathrm{D}$ revealed that that the odds of neomycin use at high frequencies was lower in cases [OR: 0.07 (0.01$0.95) ; P=0.045]$. Majority of antibacterial treatments for ear conditions included topical polymyxin B sulfate, chlorhexidine and salicylic acid. Polymyxin B suphate was prescribed in $47 \%$ of all consults (controls and cases after oclacitinib administration) that were for pruritic ears without infectious agents, and $52 \%$ of all consults for pruritic ears that had infectious agents present on an ear swab. Salicylic acid was prescribed in $20 \%$ of consults for pruritic ears without infectious agents and $11 \%$ of pruritic ears with infectious agents. Chlorhexidine was prescribed in $10 \%$ of pruritic ears without infectious agents and $6 \%$ of pruritic ears with infectious agents. The differences in use of these three antibacterials were not a significant results in the final model $(p>0.05)$.

\section{DISCUSSION}

Antimicrobial resistance (AMR) is a public health emergency and antimicrobial stewardship in veterinary practice can help reduce the pressure on the development of AMR (22). One strategy to combat the emergence of AMR in veterinary practice is to reduce, when possible, the amount of antibacterials used. Historically, empirical systemic antibacterial therapy has been the standard treatment protocol for canine skin infections associated with allergic skin disease and our data are an example of this approach to the management of both cases and controls. However, with the emergence of antibacterial resistance, topical antibacterial therapies are considered an important monotherapy or adjunct therapy (23). Therapeutic agents with potential to contribute to such reduction in use of antibacterials are important tools in the improvement of antimicrobial stewardship in veterinary medicine. Oclacitinib which is used to treat allergic dermatitis, including atopic dermatitis acts by targeting specific pathways involved in itch and inflammation. Alleviating pruritus can interrupt the itch cycle and allow for skin healing, thus aiding reduction of the chronic inflammatory changes and secondary infections (10). This study, 
TABLE 6 | Multivariable results of the association between cases and controls with respect to specific drug use.

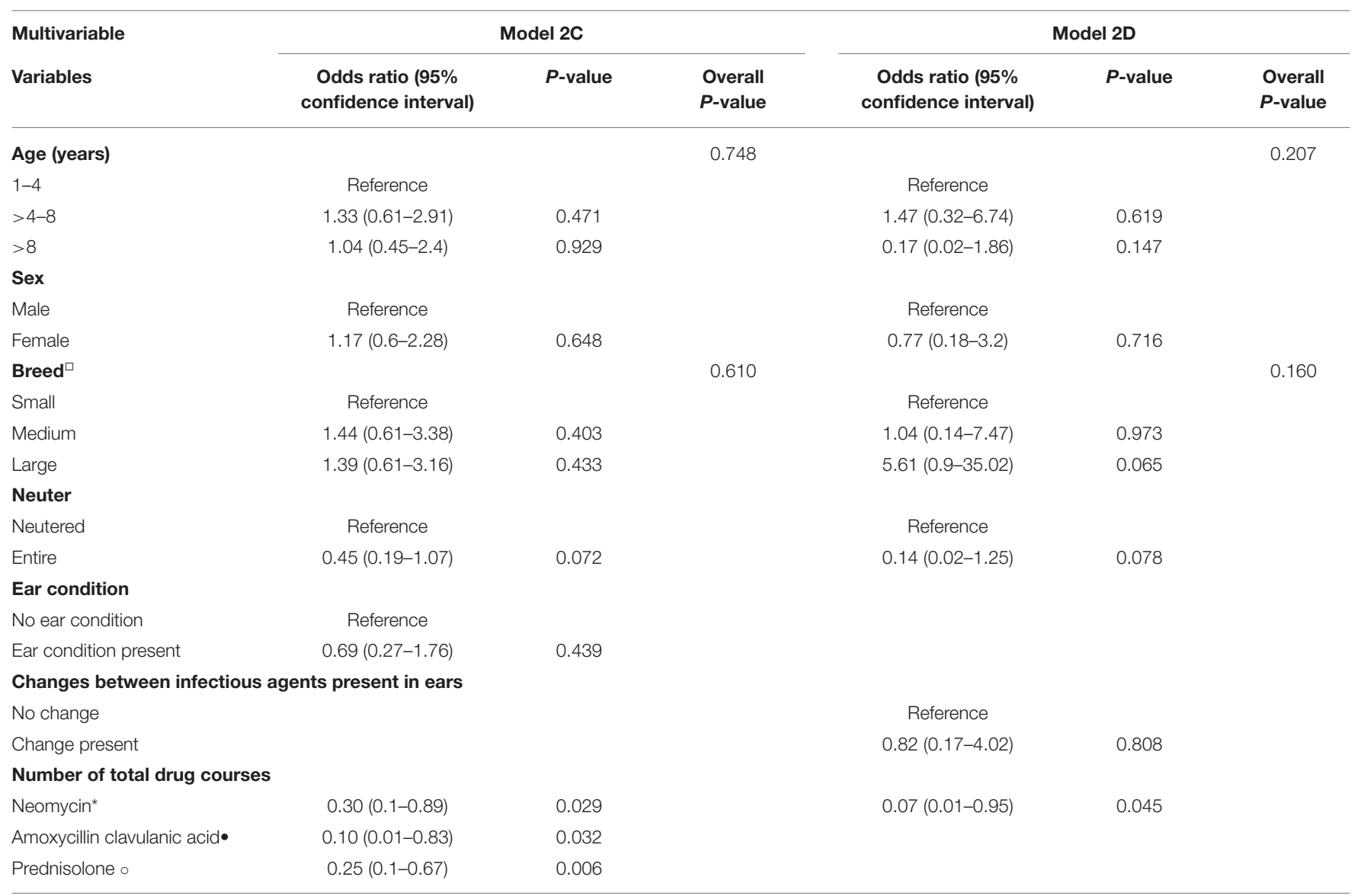

In Model $C$ drug effects were adjusted for the presence (or absence) of an ear condition and Model D drug effects were adjusted for changes between infectious agents isolated from ears. Variables with a $P<0.05$ were considered statistically significant.

${ }^{\star}$ Topical antibacterial high frequency $\geq$ once per week $\bullet$ Systemic dose $12.5-25 \mathrm{mg} / \mathrm{kg}$ every $12 \mathrm{~h}$ ○ Systemic tapering dose $0.5-1 \mathrm{mg} / \mathrm{kg} / \mathrm{day}{ }^{\square}$ Breed categories defined in Supplementary Tables $4 a, b$.

which aimed to quantify topical and systemic antibacterial use, corticosteroid use and the effect on skin categories in client-owned dogs with allergic dermatitis, including atopic dermatitis, treated with oclacitinib compared to dogs treated with other common therapies, showed that cases treated with oclacitinib had an overall reduction in their percentage usage of systemic and topical antibacterials and an improvement in their skin conditions.

In this study, cephalexin and amoxycillin clavulanic acid were the primary systemic antibacterials used in both cases and controls. Both agents are recommended as first-line antibacterials to empirically treat superficial canine pyoderma $(17,24)$. Our results revealed that dogs with allergic skin disease treated with oclacitinib had significantly $(P<0.05)$ lower odds of needing systemic antibacterials than dogs treated with other therapies. Further analysis revealed that the odds were lower specifically for amoxycillin clavulanic acid usage at $12.5-25 \mathrm{mg} / \mathrm{kg}$ every $12 \mathrm{~h}$. This result remained significant after adjusting for age, sex, breed, neuter status, presence of ear condition, skin condition at initial consult and changes between skin condition categories. We also showed that before adjusting for demographic, drug therapy, skin condition or ear condition variables, the number of cephalexin courses per animal was significantly $(P<0.05)$ reduced in dogs treated with oclacitinib. However, after accounting for important confounders in the multivariable model, the number of cephalexin courses was not significantly different between cases and controls, which could be partly explained by the dominance of cephalexin prescriptions in both groups of dogs.

In our study topical therapies represented a larger proportion of total antibacterial courses than systemic therapies in dogs receiving oclacitinib and those treated with other therapies. Overall, our univariable results demonstrate that topical antibacterial use was reduced after oclacitinib use; however, after adjustment for age, sex, breed, neuter status, presence of ear condition, skin condition at initial consult and changes between skin condition categories in the multivariable models the only significant reduction in a specific antibacterial was in topical neomycin use at a frequency of more than once a week. The use of enrofloxacin, ciprofloxacin and Polymyxin B, which are Highest Priority Critically Important Antimicrobials, were not significantly reduced, highlighting the importance of antibiotic stewardship (21). 
Glucocorticoids are another commonly used therapy to treat allergic dogs (10). Multivariable logistic regression analyses indicated that the odds of overall systemic glucocorticoid use were lower in dogs treated with oclacitinib, specifically systemic prednisolone usage at a tapering dose of $0.5-1$ $\mathrm{mg} / \mathrm{kg} /$ day. This result was retained after adjusting for age, sex, breed, neuter status, the presence of ear condition, skin conditions at baseline and changes between them. It has been suggested that the concomitant use of oral glucocorticoids with oclacitinib is discouraged due to immunosuppression concerns. Currently there are no published data demonstrating the long-term safety of concomitant use, our data suggest that veterinarians were probably inclined to avoid prednisolone use when treating with oclacitinib as only three dogs were treated with both drugs simultaneously. In contrast, twentyfour dogs from the oclacitinib treated group were prescribed 41 courses of prednisolone prior to initial oclacitinib use. The efficacy of both drugs in treating pruritic conditions have been shown to be very similar (25). The indirect reduction in glucocorticoid use could be considered beneficial due to the adverse effects associated with long-term use (10). For example, long-term glucocorticoid use in dogs has been associated with an increased risk of developing a urinary tract infection (26) and one study found urinary tract infections in $39 \%$ of dogs receiving long-term corticosteroid therapy for chronic skin diseases (27).

In addition, the results of our study also suggested that dogs treated with oclacitinib were significantly more likely to experience improvements in their skin condition categories compared to dogs treated with other therapies, which is an additional benefit of using this treatment approach. A recent study suggested that oclacitinib use had positive effects on skin barrier parameters compared to prednisolone and other treatments, such as lower transepidermal water loss (TEWL) and increased hydration (28). TEWL is an indication of total cutaneous water loss and is often used to assess skin barrier activity, as an increase in TEWL has been associated with decreased skin integrity in atopic patients (29). Perhaps the hydration levels in the skin and/or return of skin microbiome to a normal flora is associated with an improvement in skin condition but these deserve further investigation.

There are a few limitations with our study primarily associated with the nature of the clinical record data used in our investigation which need to be addressed by further research. We attempted to record the average duration of an antibacterial course, but this information was not always available, especially with topical therapeutics. Therefore, we were unable to adjust for

\section{REFERENCES}

1. Hensel P, Santoro D, Favrot C, Hill P, Griffin C. Canine atopic dermatitis: detailed guidelines for diagnosis and allergen identification. BMC Vet Res. (2015) 11:196. doi: 10.1186/s12917-015-0515-5

2. Olivry T, Deboer DJ, Favrot C, Jackson HA, Mueller RS, Nuttall T, et al. Treatment of canine atopic dermatitis: 2015 updated guidelines from the the length of antibacterial use in the final models. Information on why dogs in the control group did not receive oclacitinib was also not always available which may potentially confound some of the associations identified in this study. Details on diagnostic tests performed to confirm or rule out causes of itchy skin were not always available in the consultation notes. This was also true for diets and dietary changes in animals with food allergies as a definitive or differential diagnosis, where only eight dogs (one case and seven controls) experienced dietary changes. While we did not include the terms "otitis" and "pododermatitis" - given that these are not part of on label conditions-our search yielded some cases that had these conditions listed in clinical records. This indicates that oclacitinib is potentially being used with some success for a wider list of allergic dogs, the impact of which needs further investigation. In addition the effects noted are associations and better causal relationships could be ascertained using a prospective study design such as an intervention trial to confirm the associations noted in this study.

In conclusion, dogs with allergic skin disease treated with oclacitinib had a significantly reduced use of systemic and topical antibacterials and significantly higher odds of experiencing improvements in their skin condition category.

\section{DATA AVAILABILITY STATEMENT}

The raw data supporting the conclusions of this article will be made available by the authors, without undue reservation.

\section{AUTHOR CONTRIBUTIONS}

HR conducted the study, completed the analysis, and drafted the manuscript. JG, EM, and RS were involved in the study design and finalization of the manuscript. All authors contributed to the article and approved the submitted version.

\section{FUNDING}

This research was possible thanks to a research grant under the University of Queensland Science with Impact Fund with partial funding provided by the industry partner Zoetis Australia, Pty Ltd.

\section{SUPPLEMENTARY MATERIAL}

The Supplementary Material for this article can be found online at: https://www.frontiersin.org/articles/10.3389/fvets. 2021.631443/full\#supplementary-material

3. Linek M, Favrot C. Impact of canine atopic dermatitis on the health-related quality of life of affected dogs and quality of life of their owners. Vet Dermatol. (2010) 21:456-62. doi: 10.1111/j.1365-3164.2010.00899.x

4. Noli C, Minafò G, Galzerano M. Quality of life of dogs with skin diseases and their owners. Part 1: development and validation of a questionnaire. 
Vet Dermatol. (2011) 22:335-43. doi: 10.1111/j.1365-3164.2010. 00954.x

5. Olivry T, Bizikova P. A systematic review of randomized controlled trials for prevention or treatment of atopic dermatitis in dogs: 2008-2011 update. Vet Dermatol. (2013) 24:97-117.e125e116. doi: 10.1111/j.1365-3164.2012.01088.x

6. Anturaniemi J, Uusitalo L, Hielm-Björkman A. Environmental and phenotype-related risk factors for owner-reported allergic/atopic skin symptoms and for canine atopic dermatitis verified by veterinarian in a Finnish dog population. PLoS ONE. (2017) 12:e0178771. doi: 10.1371/journal.pone.0178771

7. Layne EA, Moriello KA. What's new with an old problem: drug options for treating the itch of canine allergy. Vet Med. (2015) 110:103-10

8. Moyaert H, Van Brussel L, Borowski S, Escalada M, Mahabir SP, Walters $\mathrm{RR}$, et al. A blinded, randomized clinical trial evaluating the efficacy and safety of lokivetmab compared to ciclosporin in client-owned dogs with atopic dermatitis. Vet Dermatol. (2017) 28:593-e145. doi: 10.1111/vde.12478

9. Santoro D, Marsella R, Pucheu-Haston CM, Eisenschenk MNC, Nuttall T, Bizikova P. Review: pathogenesis of canine atopic dermatitis: skin barrier and host-micro-organism interaction. Vet Dermatol. (2015) 26:84e25. doi: 10.1111/vde. 12197

10. Cosgrove SB, Wren JA, Cleaver DM, Martin DD, Walsh KF, Harfst JA, et al. Efficacy and safety of oclacitinib for the control of pruritus and associated skin lesions in dogs with canine allergic dermatitis. Vet Dermatol. (2013) 24:479-e114. doi: 10.1111/vde.12047

11. Olivry T, Deboer DJ, Favrot C, Jackson HA, Mueller RS, Nuttall T, et al. Treatment of canine atopic dermatitis: 2010 clinical practice guidelines from the International Task Force on Canine Atopic Dermatitis. Vet Dermatol. (2010) 21:233-48. doi: 10.1111/j.1365-3164.2010.00889.x

12. Mason IS, Lloyd DH. The role of allergy in the development of canine pyoderma. J Small Anim Pract. (1989) 30:2168. doi: 10.1111/j.1748-5827.1989.tb01540.x

13. Deboer DJ, Marsella R. The ACVD task force on canine atopic dermatitis (XII): the relationship of cutaneous infections to the pathogenesis and clinical course of canine atopic dermatitis. Vet Immunol Immunopathol. (2001) 81:239-49. doi: 10.1016/S0165-2427(01)00345-2

14. Pierezan F, Olivry T, Paps JS, Lawhon SD, Wu J, Steiner JM, et al. The skin microbiome in allergen-induced canine atopic dermatitis. Vet Dermatol. (2016) 27:332-82. doi: 10.1111/vde.12366

15. Mcewan NA, Mellor D, Kalna G. Adherence by Staphylococcus intermedius to canine corneocytes: a preliminary study comparing noninflamed and inflamed atopic canine skin. Vet Dermatol. (2006) 17:151-4. doi: 10.1111/j.1365-3164.2006.00503.x

16. Fazakerley J, Nuttall T, Sales D, Schmidt V, Carter SD, Hart CA, et al. Staphylococcal colonization of mucosal and lesional skin sites in atopic and healthy dogs. Vet Dermatol. (2009) 20:179-84. doi: 10.1111/j.1365-3164.2009.00745.x

17. Hillier A, Lloyd DH, Weese JS, Blondeau JM, Boothe D, Breitschwerdt E, et al. Guidelines for the diagnosis and antimicrobial therapy of canine superficial bacterial folliculitis (Antimicrobial Guidelines Working Group of the International Society for Companion Animal Infectious Diseases). Vet Dermatol. (2014) 25:163-43. doi: 10.1111/vde.12118
18. Gonzales AJ, Bowman JW, Fici GJ, Zhang M, Mann DW, Mitton-Fry M. Oclacitinib (APOQUEL $($ ) is a novel Janus kinase inhibitor with activity against cytokines involved in allergy. J Vet Pharmacol Ther. (2014) 37:31724. doi: $10.1111 /$ jvp. 12101

19. Pickup E, German A, Blackwell E, Evans M, Westgarth C. Variation in activity levels amongst dogs of different breeds: results of a large online survey of dog owners from the UK. J Nutr Sci. (2017) 6:e10. doi: 10.1017/jns. 2017.7

20. The Kennel Club. Breed Information Centre. (2018). Available online at: https://www.thekennelclub.org.uk/services/public/breed/Default.aspx

21. World Health Organization. Critically important antimicrobials for human medicine, 6th revision (2019). Licence: CC BY-NC-SA 3.0 IGO

22. World Organisation for Animal Health (2018). Available online at: http:// www.oie.int/en/for-the-media/amr/ (accessed October 25, 2018).

23. Bajwa J. Staphylococcal pyoderma: challenges and therapeutic considerations. Vet Rec. (2018) 182:431-3. doi: 10.1136/vr.k1563

24. Beco L, Guaguère E, Méndez CL, Noli C, Nuttall T, Vroom M. Suggested guidelines for using systemic antimicrobials in bacterial skin infections: part 2- antimicrobial choice, treatment regimens and compliance. Vet Rec. (2013) 172:156-60. doi: 10.1136/vr.101070

25. Gadeyne C, Little P, King VL, Edwards N, Davis K, Stegemann MR. Efficacy of oclacitinib (Apoquel ${ }^{\circledR}$ ) compared with prednisolone for the control of pruritus and clinical signs associated with allergic dermatitis in clientowned dogs in Australia. Vet Dermatol. (2014) 25:512-86. doi: 10.1111/vde. 12166

26. Torres SM, Diaz SF, Nogueira SA, Jessen C, Polzin DJ, Gilbert SM, et al. Frequency of urinary tract infection among dogs with pruritic disorders receiving long-term glucocorticoid treatment. J Am Vet Med Assoc. (2005) 227:239-43. doi: 10.2460/javma.2005.227.239

27. Ihrke PJNAL, Ling GV, Stannard AA. Urinary tract infection associated with long term corticosteroid administration in dogs with chronic skin diseases. $J$ Am Vet Med Assoc. (1985) 186:43-6.

28. Marsella R, Ahrens K, Wilkes R, Trujillo A, Dorr M. Comparison of various treatment options for canine atopic dermatitis: a blinded, randomized, controlled study in a colony of research atopic beagle dogs. Vet Dermatol. (2020) 31:284 doi: 10.1111/vde.12849

29. Cobiella D, Archer L, Bohannon M, Santoro D. Pilot study using five methods to evaluate skin barrier function in healthy dogs and in dogs with atopic dermatitis. Vet Dermatol. (2019) 30:121-e134. doi: 10.1111/vde. 12723

Conflict of Interest: All authors report grants from Zoetis Australia, Pty Ltd, during the conduct of the study.

Copyright (c) 2021 Rynhoud, Gibson, Meler and Soares Magalhães. This is an openaccess article distributed under the terms of the Creative Commons Attribution License (CC BY). The use, distribution or reproduction in other forums is permitted, provided the original author(s) and the copyright owner(s) are credited and that the original publication in this journal is cited, in accordance with accepted academic practice. No use, distribution or reproduction is permitted which does not comply with these terms. 